\title{
Oxygen-ozone therapy in the treatment of functional overload syndromes of music professionals
}

\author{
Gaetano Cuccio, ${ }^{1,2}$ Marianno Franzini, ${ }^{2}$ Luigi Valdenassi ${ }^{2,3}$ \\ ${ }^{1}$ Doctor-Professor of Piano at the Conservatory of Music V. Bellini of Palermo, Institute of Advanced Training in \\ Artistic, Musical and Coreutic AFAM-MIUR, Palermo; ${ }^{2}$ Oxygen-Ozone Therapy Scientific Society, Gorle (BG); \\ ${ }^{3}$ Department of Internal Medicine and Medical Therapy, University of Pavia, Pavia, Italy
}

\begin{abstract}
Musculoskeletal disorders of musicians and entertainment professionals in general have been the subject of studies and research since the nineteenth century.

For the physician, they represent a grouping of pathologies that are easy to diagnose but difficult to cure, especially due to the high frequency of relapses. Oxygen-ozone therapy is an effective method for the treatment of these illnesses without the side effects of other drugs.
\end{abstract}

\section{Introduction}

Therapy with Oxygen-Ozone mixtures is frequently used for the treatment of illnesses linked to the musculoskeletal system. In entertainment professionals, it is used for the effective metabolic action that it performs in tissue healing processes. These conditions sometimes cause diagnostic and therapeutic difficulty due to the complexity of factors that contribute to the formation of the lesion.

Musicians are considered a category with a high occupational risk for functional overload disorders. ${ }^{1,2}$

Correspondence: Gaetano Cuccio, Oxygen-Ozone Therapy Scientific Society, via Don L. Sturzo 2, 24020 Gorle (BG), Italy.

Tel.: +39.035.19910105.

E-mail: info@ossigenoozono.it

Key words: Oxygen-ozone therapy; tendon; oxidative stress; vascularization; healing.

Conflict of interest: the authors declare no potential conflict of interest.

Received for publication: 13 December 2017.

Accepted for publication: 21 December 2017.

CCopyright G. Cuccio et al., 2017

Licensee PAGEPress, Italy

Ozone Therapy 2017; 2:7246

doi:10.4081/ozone.2017.7246

This article is distributed under the terms of the Creative Commons Attribution Noncommercial License (by-nc 4.0) which permits any noncommercial use, distribution, and reproduction in any medium, provided the original author(s) and source are credited.
These frequently slow down the artist's activity, limiting his/her career. Among the main causes, we would mention the excessive workload due to the great demand of the entertainment sector and the continuous repetitive actions that musicians have to carry out in order to produce sounds. In time, this causes repeated micro-traumas and strong oxidative stresses to the anatomical structures involved.

\section{Biomechanical trauma and stress}

Biomechanical stress occurs in all categories of musicians. The vocal system of singers, mainly opera singers, is subjected to the breakdown of the vocal structures with a wear and tear of the cricoarytenoid and aricorniculate articulations, while in oboists, as for all single or double reed instrument players and basically for all aerophone instruments, the prolonged use of pulmonary air flow, subjected to continuous exertions and apneas, to modulate emission and pressure of the breath in order to effectively vibrate the reed, leads to denaturation processes of the elastic fibers, intervening in the pathogenesis of asthma from the stress.

In trumpet players, the microtrauma generated by the vibrations of the upper lip, used as a vibratory membrane, determines the early loss of the incisors. In violin players, vibrations with different intensities and frequencies transmitted by the sound box on the neck can cause carotid arteritis.

During the artists' formative period, the improper use of instrumental techniques causes the ideal conditions for the onset of pathologies linked to muscles and tendons. This is also due to theinadequate knowledge of the principles of the physiology of movement ${ }^{3}$ by the musician, teacher and student.

\section{Epidemiology}

Epidemiological studies show that the musician belongs to one of the categories with the greatest risk of contracting illnesses affecting the muscular - skeletal ${ }^{3,4}$ system.

These include functional overload syndromes, nerve compression and focal dystonia.

Over-use syndromes include a set of musculoskeletal disorders and inflammatory, traumatic and degenerative diseases, affecting muscles, tendons and the adjoining gliding structures. ${ }^{3}$

The greater incidence of injuries due to repetitive mechanical stress is observed in the upper and lower limbs, even if fractures, muscular lesions, osteo-tendon detachments and large inflammatory syndromes prevail in the latter. ${ }^{4}$ 
Pain in the thoracic body may present itself as transient, acute, recurrent, persistent or chronic, also manifesting itself after a traumatic incident. It is located predominantly in the region corresponding to the proximal or distal insertion of the tendons of the flexor and extensor muscles of the arm, forearm, shoulder, leg, but also all along the tendon.

There is a close relationship between the onset of musculoskeletal disorders and instrument playing. For entertainment professionals, the workload consists of several hours of practice, study, auditions and daily performances, with a greater probability of developing noxae in relation to the long-term muscle activity. ${ }^{4}$

Illnesses that affect the hand are rhizarthrosis and tenosynovitis, pathologies that mainly affect clarinet, oboe and English horn players. They are probably also caused by the weight of the instrument that the musician must hold with his/her thumb. ${ }^{5}$

\section{Mechanics of movement}

The difficulties that physicians must face in order to draw a complete and detailed clinical picture of the functional pathologies afflicting the musician sometimes require specific knowledge on the instrument's technical aspects. ${ }^{5}$

In the body, the freedom of movement of the kinematic chains is a consequence of infinite position combinations and variations that are accessible to these chains. ${ }^{6}$

In particular, the extremities make contact with surfaces that change shape instantly (strings, keyboards, etc.).

The individual kinematic chains of the limb, such as the shoulder, arm, forearm and hand, are all placed on the same plane, placed perpendicularly to the contact line represented by the keyboard (piano etc.) ${ }^{6}$ or parallel to the strings (harp etc.).

They adapt to changes in the state of the mechanical parts, such as weight and elasticity, with movements that are the expression of very rapid muscular arrangements that the artist processes in fractions of a second.

To describe the problems that playing the violin causes, one can see how the violinist is forced to take on uncomfortable and sometimes unnatural positions in order to produce the sound. This allows him/her to coordinate the movements with great precision between the bow and fingers that, moving along the fingerboard with great agility, modulate strength, speed and pressure according to the quantity and quality of the sound. ${ }^{7}$

The musician moves the bow with the right limb, placing the arm in abduction, the forearm in pronation supination and the elbow sideways, while with the left limb he/she rotates the arm around the axis, flexes the forearm in pronation and supersedes the elbow, forcing the hand to take on the ideal form in order to come in contact with the strings. These complications actually facilitate a perfect motor coordination between both arms, however forcing the muscles to carry out frequent dyssynergic contractions (Figure 1). ${ }^{8}$

\section{Etiopathogenesis}

Action as a consequence of the use of force to the bones is controlled by the neuromuscular system. ${ }^{9}$

The movements generated by the muscular contraction, in the kinematic chains, can be represented graphically by vectors characterized by intensity, course and direction. ${ }^{10}$

Considering the major axis of the muscular soma and of the long tendon to which the exertion is transmitted, we observe that both are oriented on the same plane and converging on the points of bone insertion. Movement is the result of forces and resistances as a dynamic compromise between extrinsic and intrinsic factors, including among the former the alteration of vascularization and the tendinous array, aging, metabolic diseases and psychophysical factors, while among the latter resistances and weight, body mass, biomechanical factors, friction, temperature, pressure of certain areas, humidity and acoustics of the locations. ${ }^{11}$

The pathogenetic hypothesis that mechanical stress is one of the main factors in the onset of muscoloskeletal disorders of over-use in musicians, as well as in athletes, could justify a relationship between cause and effect. ${ }^{12}$ In physics, the human body is considered an open thermodynamic system that is able to exchange energy with the environment, abruptly adapting to changes of the environment. If we look at the body of an athlete running or of a dancer during a performance, we can say, regarding the system body, that this system during the action of motion, will undergo a variation of the quantity of mechanical energy (en. Kinetics + en. Potential) not null and void but equal to the work done by the conservative forces (frictions), or extrinsic factors, which generate dissipation of energy..$^{13}$

In kinematic systems, which are the individual anatomical segments of the limbs (shoulder, arm, forearm, hand, etc.), the amount of energy produced varies over time and is never equal to zero. The work produced by the individual systems, as a result of the force generated by the agonist and antagonist muscles, when it differs from the balance between the extrinsic and intrinsic factors, could undergo, during the sudden accelerations or decelerations, a variation of direction with a different angle of application.

A small part of the mechanical energy is dissipated from the body to the environment (for example in the form of heat), but a large part will be stored and then discharged, according to the principles of thermodynamics. If the energy generated is different from the internal mechanical balances, it could deform the anatomical structures thus injuring them. ${ }^{14,15}$

The tendon tissue, stressed with loads greater than its resistance, undergoes strong structural variations. The fibrils arranged in parallel and bundled together have a wavy pattern with periodic changes of direction. The collagen fibers thus organized, known as crimps, form triangular geometrical figures of variable sizes.

They have angles of different widths in order to ensure, with their deformation, the resistance and elasticity of the tendon, during muscle contraction.

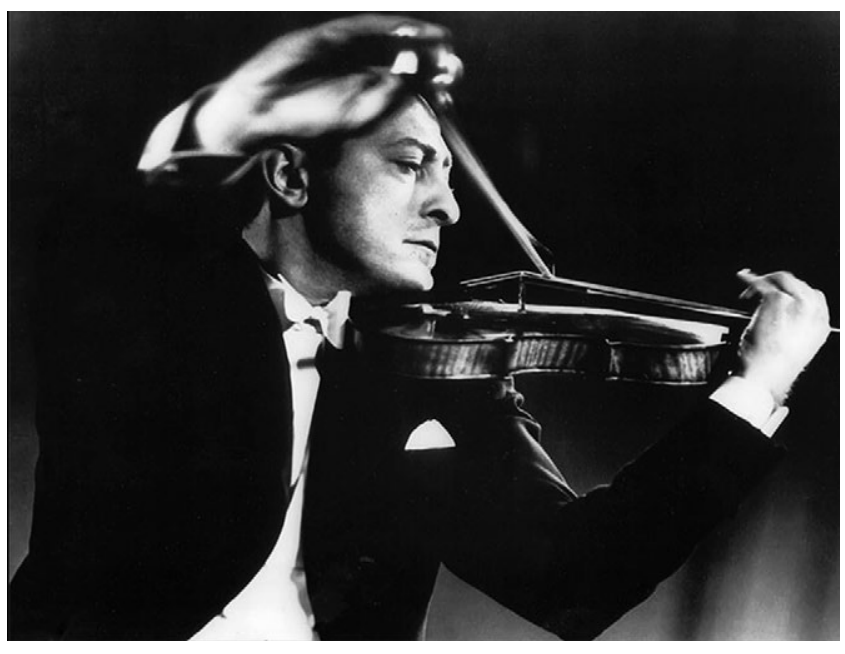

Figura 1. J. Heifetz (1941) position of the limbs on the violin. 
The tendon tissue preserves the integrity of the microstructure with elongations lower than $4 \%$ of the length, while with greater distensions, partial or complete breaks of the collagen bundlesare generated (Figures 2 and 3).

\section{Pain in entertainment professionals}

Pain can arise in any body part that is subjected to repeated trauma caused by intense artistic activity or athletic training.

In dancers as well as in athletes, where entire muscle chains manage moving body masses, ${ }^{16}$ the symptoms can be excessive with injuries, even traumatic, which can affect tendons (tendonitis, rupture degeneration), tendon insertions (detachment), muscular soma (tearing and rupture), joints (rupture, wear/tear, hemarthros, dislocations), bones (fractures).

The clinical picture is characterized by pain, inflammation and swelling of the part with motor difficulty, which in artists evolves prematurely in functional impotence.

Pain can be transient or continuous, without evident damage to the tissue but which ceases with the pathogenic incident. Nociceptive, of short duration, with relationship of cause and effect, persistence of the damage and regression with repair. It is recurrent when the harmful ailment persists but is extinguished with the healing of the lesion. Chronic when it is characterized by persistent trauma and is associated with deep-set changes in the musician's personality and lifestyle.

\section{Vascularization of the tendon}

The tendon is a poorly vascularized tissue. The vessels follow a sinuous path reaching the tendon through the myotendinous, osteotendinous, or paratenon ${ }^{17}$ insertion points. The vascular network is not uniform, with variable densities in relation to specific functions. The condition of hypovascularization refers to the presence of antiangiogenic factors or their inhibition. The vascular endothelial growth factor of proangiogenic protein (VEGF), as well as endostatin also involved in vascularization processes, is poorly expressed in intact tendons of adults while it is present in injured tendons. ${ }^{18}$

The condition of hypoperfusion may be associated with degeneration or tendon rupture, although it does not seem to be a primary cause. ${ }^{19}$

In achilles tendinopathy, cytokines such as VEGF, epidermal growth factor (EGF) and platelet-derived growth factor (PDGF) are over expressed. VEGF promotes angiogenesis but also regulates the expression of MMPs by reducing the levels of tissue inhibitors for metalloproteases (TIMP-3) present during remodeling repair processes of the tendon tissue. ${ }^{19,20}$ Little attention has been paid to the lymphatic system which carries out important drainage functions in tissue repair phases. ${ }^{19}$ The microcirculation intervenes in the repair processes of the tendons and ligaments, also favoring the best clearance of the metabolites. ${ }^{18}$ Renowned research has shown the relationship between the tendon lesions and the degeneration of the tendinous matrix, in which there are structural and necrobiotic alterations due to inflammatory and enzymatic degradation processes prior to the trauma. ${ }^{21}$ Chronic tendon injury is characterized by pain and functional impotence, showing intense vascularization and cell proliferation. It may happen that, in the preclinical period, less tissue perfusion may occur with reduced vascular expression and metabolic exchanges. This hemodynamic picture is probably one of the decisive factors of degeneration of the tendon tissue. During the repair processes there is an increase in the cell population and the quantity of the vessels. ${ }^{22}$ In this phase, elements such as monocytes, leucocytes and macrophages are responsible for the synthesis of angiogenic factors such as fibroblast growth factor (bFGF), plateletderived growth factor, epidermal growth factor and transforming growth factor (TGF-beta1). In the repair process the vascular endothelial factor or basic epidermal growth factor (BEGF) was pinpointed in tendon trauma injuries, acting as a regulator of integrin expression aV beta 3 and a5 beta 1 in the tenocytes. ${ }^{19}$ The initial lesion does not always produce a vascular or fibroproliferative response. Hypoxia caused by reduced perfusion, even in the absence of inflammation and pain, seems to be one of the main factors of degeneration and subsequent rupture of the tendon or ligament (1918). The vascular response also begins when the lesion occurs in an already perfused area. The VEGF factor, in such a scenario of events, is activated by hypoxia and ischemia, relating pathogenic and angiogenic factors. ${ }^{19-22}$

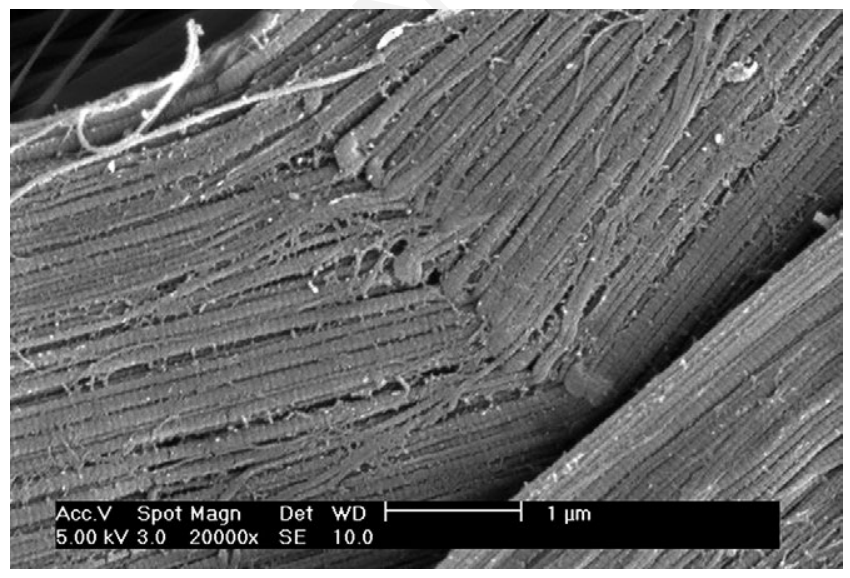

Figura 2. SEM: single tendon crimp with straight and parallel fibrillar segments joined by knots (fibrillar crymps). From the thesis of Dr. Maria Maciocca, PhD, University of Bologna, Faculty of Motor Sciences.

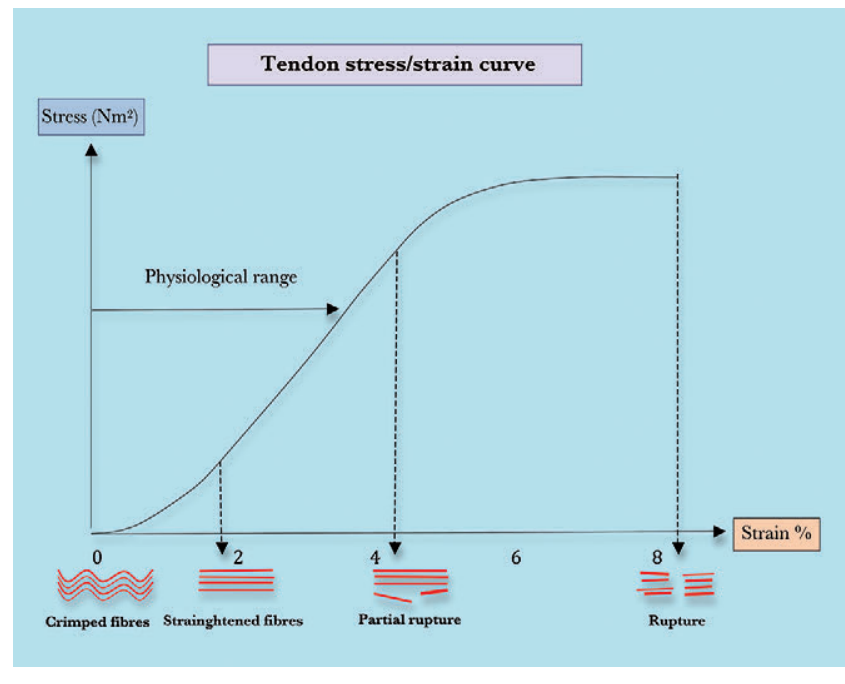

Figure 3. Voltage/deformation curve. 


\section{Muscle activity and oxidative stress}

Intense muscle activity is known to generate high levels of reactive oxygen species (ROS) in skeletal muscles. ${ }^{23}$ The greater production of free radicals is mainly caused by the altered transport of electrons in the mitochondria, due to the excessive increase in oxygen consumption and the synthesis of NADPH-oxidase during the ischemia and reperfusion phases of muscles and internal organs. ${ }^{24}$ These molecules cause irreversible damage to DNA, proteins and lipids, also acting as mediators in cell metabolism by stimulating the synthesis of antioxidants (redox system). ${ }^{25}$ ROSs are unstable molecules and ions, very reactive due to an unpaired electron. In muscle cells, the potential producers of free radicals are the mitochondria and the species derived from nitrogen (RNS), the nicotinamide adenine dinucleotide phosphate (NADPH) and the phospholipases A2 (PLA2), xanthine oxidase (XO) and lipoxygenase. Physical exercise produces large quantities of free radicals whose actions and effects depend on concentration, exposure time and exercise routine. Radicals produced in excessive quantities may not be effectively buffered byendogenous ${ }^{25}$ antioxidants (Figure 4).

This can cause severe oxidative damage to protein and DNA structures, lipid peroxidation, mitochondrial dysfunction, apoptosis tissue necrosis involving the adenosine triphosphatase (ATPase), the contractile proteins and mitochondrial ${ }^{25}$ enzymes in this mechanism. Moderate exercise results in increased antioxidant capacity, promotes mitochondrial biogenesis, improves insulin sensitivity and cytoprotection. Regular exercise facilitates the respiratory activity of the skeletal muscle..$^{25,26}$ The superoxide dismutase (SOD), catalase (CAT) and glutathione peroxidase (GPX) antioxidant enzymes, as well as the non-enzymatic ones, including $\alpha$-tocopherol and vitamin $\mathrm{C}, \beta$-carotene and coenzyme Q10, lipoic acid and glutathione (GSH), cysteine, uric acid and flavonoids, represent the main defense against free radicals produced during the year. ${ }^{23-25}$ Recent study has identified in thioredoxin reductase2 (TxnRd2) one of the key factors for mitochondrial $\mathrm{H} 2 \mathrm{O} 2$ reduction in skeletal muscle. ${ }^{25}$

The high oxidative stress produced by intense exercise as well as traumatic injuries activates the nuclear transcription factor kappa $\beta(\mathrm{NFk} \beta)$ triggering the inflammation processes in tissues as well as the production of cytokines, COX2, PGE2. On the other hand, moderate exercise generates reduced oxidative stress and the activation of the erythroid nuclear factor $2(\mathrm{Nrf} 2)$ which modulates the transcription for the synthesis of the elements of the antioxidant response (ARE). ${ }^{24}$

Muscle injuries, in damaged tissues, are also caused by the activation of the cell-mediated immunity elements. They release different cytokines such as interferonY (IFN-Y), interleukin-1 (IL$1)$, IL- 8 and the tumor necrosis factor $\alpha(\mathrm{TNF}-\alpha)$. Muscle damage caused by excessive physical exercise has been associated with the degradation of myofilaments of sarcomeres and sarcolemma, loss of integrity of myofibrils and acute inflammatory response, with diminished contractile capacity of the muscles. The adaptation to muscular exertion and resistance that occurs after a trauma follows the classic sequence of restorative phenomena: damage, inflammation and regeneration. ${ }^{26}$ In the first phase of the tissue repair process, the mechanisms of inflammation are activated. In the muscle, just before injury, high concentrationsof IL-1, IL-6 and IL10 were found. Among these IL-6 was classified as a pro and antiinflammatory cytokine. It acts primarily as one of the anti-inflammatory factors of the acute phase, inhibiting the inflammation mediated by TNF- $\alpha$ and IL-1. ${ }^{27}$ Anti-inflammatory cytokines IL10 suppress the production of TNF- $\alpha$, IL-1, IL- 6 , IL- 8 and of the granulocyte colony stimulating factor (G-CSF) reducing plasma levels and intervening in the stages of restoration and recovery of the tendon. ${ }^{28}$

\section{Modeling, remodeling and healing of tendon tissue}

Tendon pathologies sometimes present themselves with clinical pictures characterized by the absence of a specific relationship between symptomatology and organ damage. The acute rupture of the tendon may also not be preceded by any sign of suffering. The healing process of acute, chronic and degenerative injuries involves some phases in succession. ${ }^{29}$ Intrinsic and extrinsic factors intervene in the occurrence of traumas. Basically, extrinsic factors prevail in acute trauma, while both factors act in chronic injuries. ${ }^{30}$

Among the intrinsic ones we cite the absence of fibrillar alignment and biomechanical errors, while among the extrinsic ones, the excessive movement under load, continuous and repetitive, as occurs during intense athletic exercise or ballet dancing. Muscular surmenage generates intense oxidative stress and continuous microtraumas. The body reacts by activating repair mechanisms, initiating processes classified in texts as modeling, remodeling and regeneration phases. The attempt to repair the tendon, ligament or adnexae is insufficient if the continuous trauma and the oxidative damage exceed the regenerative capacity of the tissue.

Today, there is still little familiarity with the pathogenesis of

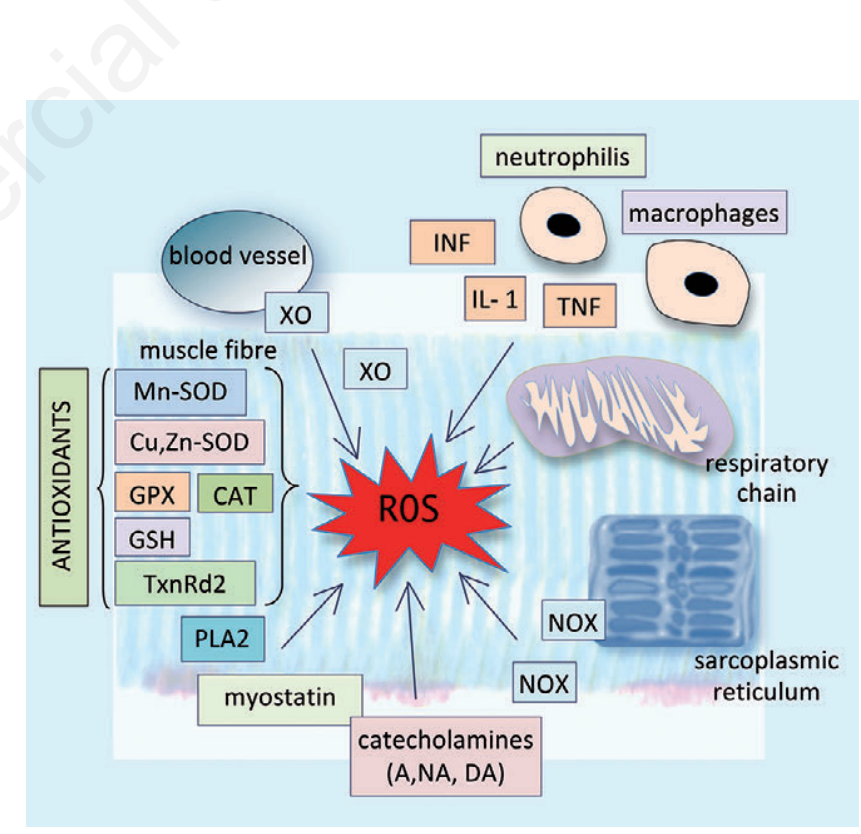

Figure 4. Sources of reactive oxygen species (ROS) and endogenous antioxidants in skeletal muscle fibers. Excessive exercise produces large amounts of free radicals, while moderate exercise leads to more endogenous antioxidants that neutralize ROS. Various metabolic processes and elements of mediated cell immunity such as neutrophils, monocytes and activated macrophages are involved in production. A, adrenaline; CAT, catalase; $\mathrm{Cu}, \mathrm{Zn}$ SOD, copper-sulphur superoxide dismutase; DA, dopamine; GPX, glutathione peroxidase; GSH, glutathione; IFN, interferon; IL-1, interleukin-1; Mn-SOD, manganese superoxide dismutase; NA, noradrenaline; NOX, nicotinamide adenine dinucleotide phosphate oxidase; PLA2, phospholipase A2; SR, sarcoplasmic reticule; TNF, tumor necrosis factor; $T \times n R d 2$, thioredoxin reductase 2; $\mathrm{XO}$, xanthine oxidase. 
tendinopathies due to the complexity of the processes involving numerous factors including hypoxia, ischemia, oxidative stress, hyperthermia, uncontrolled cell apoptosis, factors of inflammation, degradation of the matrix.

The tendinous affection in its evolution sometimes presents clinical pictures of disordered healing, even in the absence of inflammation or degenerative processes of the tissue. ${ }^{30}$ Pain related to inflammation can also be caused by mechanical and biochemical factors. ${ }^{22}$ In tissues that are subject to degenerative phenomena, a greater expression of pro-inflammatory prostaglandin PGE2 has been observed, also in the first part of healing, in which neutrophils, monocytes and macrophages migrate to the site of the lesion with intense phagocytic activity. They release vasoactive and chemotactic factors on site, stimulating vascular permeability and angiogenesis, collagen and glycosaminoglycan neosynthesis, initiating remodeling processes, with the formation of healing tissue. In this phase, the quantity of cellular elements and the synthesis of collagen are reduced, and the tendons are consolidated and matured. ${ }^{30}$ The metabolism of the tenocytes remains high and the collagen fibers are organized to resist the forces generated by muscle contraction. Tenoblasts also proliferate and migrate during repair processes in which protein modulators with enzymatic activity such as collagenase-3, growth factors and various cytokines also intervene. Growth factors stimulate the formation of vessels, the proliferation of fibroblasts, of the tenocytes and the production of collagen. In the remodeling and healing processes, nitric oxide (NO) acts in the angiogenetic processes and in the vessel dilation. ${ }^{31}$ Nitroxide stimulates the synthesis of collagen by regulating the growth factor beta-1 (TGF-b1) also modulating other cytokines such as the growth factor of the basic fibroblasts (bFGF), the nuclear transcription factor $\mathrm{K}$ beta $(\mathrm{NF}-\mathrm{K} \beta)$ and the insulin growth factor (IGF). ${ }^{32,33}$

\section{Growth factors and healing of the tendon}

Growth factors intervene during all tendon and ligament repair processes.

The healing of a tendon can be outlihned in three partially overlapping phases: inflammatory phase of fibroplasia and remodeling. ${ }^{34}$

In the damaged tissue the blood floods the lesion and the platelets come in contact with the uncovered collagen by aggregating and forming a clot that takes on specific functions. It acts as a supporting structure allowing cells to migrate, proliferate and form protein structures. In this phase, platelets release growth factors, mainly PDGF, TGF- $\alpha$ and TGF- $\beta$, while vascular endothelial growth factor (VEGF) binds to VEGF-R2 angiogenesis receptors expressed on angioblasts, stimulating the formation and proliferation of endothelial cells. Neovascularization is also controlled by angiopoietins (ANG1-2) that promote NO synthesis, dilatation and vessel lumen formation. When a cell lacks oxygen it starts the synthesis of the transcription factor HIF, or hypoxia-inducible factor, which stimulates the synthesis of VEGF which in turn regulates the tyrosine-kinase activity favoring the processes of capillary germination in angiogenesis. From the beginning of the tendon repair processes, the FGF fibroblast growth factor works by facilitating the migration and proliferation of endothelial cells, intervening on the production of collagen and formation of fibrils. It participates during inflammation with the stimulation of angiogenesis, cell differentiation and fibroplasia. The PDGF, released from the platelets thanks to the chemotactic properties, draws out and regulates the action of macrophages and fibroblasts, facilitating the fibroplasia and long-term remodeling of the maturation phase. Also the trans- forming growth factor beta (TGF- $\beta$ ) acts on the remodeling of the matrix by organizing the structure and density of the collagen fibers, regulating cell behavior in the different healing phases of recovery. ${ }^{34,35}$ In the initial part of the repair phase, the derived growth factor of the PDGF platelets also regulates the expression ofinsulin-like growth factor (IGF-1), which in addition to accelerating cell proliferation supports the synthesis of collagen and other ECM components during the remodeling phase. ${ }^{22,34,35}$ TGF- $\beta$ is produced by most of the cells involved in the healing processes, remaining high during inflammation just before the traumatic incident. One of the growth factor isoforms TGF- $\beta 1$ is responsible for the formation of scar tissues differently from the TGF- $\beta 3$ isoform, which acts as a negative regulator on scarring processes. The transformation of the growth factor $\beta$ (TGF- $\beta$ ) also serves to regulate the synthesis of collagen during physical exercise. ${ }^{29,34}$

The pro-inflammatory mediators produced by macrophages, lymphocytes and fibroblasts as well as the interluchine-2 (IL-2), interferon - $\gamma$ (INF- $\gamma$ ), Tumor Necrosis Factor- $\beta$ (TNF- $\beta$ ) tumor necrosis factor (TNF- $\alpha$ ), promote and regulate the mechanisms linked to inflammation directly or indirectly. They stimulate the activation of macrophages while the Th2 lymphocytes that produce IL4, IL-5, IL-6 and IL-10, seem to inhibit its action. Macrophages involved in repair processes are classified as M1 and M2 phenotypes in relation to receptors expression. M1 Macrophages produce larger amounts of pro-inflammatory cytokines such as IL-12 and TNF- $\alpha$, while M2 macrophages express large quantities of IL-10 and TGF$\beta$, inhibiting the release of pro-inflammatories. Metalloproteases also have important functions during tissue remodeling by acting on the matrix. ${ }^{22,30,34,35}$ After the trauma, in the first two weeks, we can observe a greater synthesis of MMP-9 and MMP-13 (collage nasi3 ), involved in the processes of degradation of collagen, while the levels of MMP-2, MMP-3 and MMP-14 (MP1-MMP) ${ }^{21}$ remain high, even subsequently.

Hyaluronic acid promotes collagen deposition and cellular activity during matrix formation. Polyphosphates glycosaminoglycans also intervene in the collagen maturation and grouping processes by regulating the diameter and density of the fibrils. In this phase, the neoformation of the vessels ensures blood circulation of the tissue favoring metabolic exchanges.

\section{Oxygen-ozone therapy}

Oxygen-ozone therapy uses mixtures of $\mathrm{O} 2 \mathrm{O} 3$ in different concentrations.

This model of therapy is known and practiced on vascular and immune pathologies. ${ }^{36}$ Institutes and clinics all over the world adopt oxygen-ozone in their protocols as a treatment for various diseases or as an adjuvant treatment of other therapies, including oncological ones. The metabolic effects of ozone depend on the action that this exerts on the three lines of glucose, protidic and lipid metabolism. Although not a free radical, it behaves like an oxidizing substance, generating a controlled oxidative response. ${ }^{24,36}$

Ozone, a triatomic form of oxygen, is an unstable molecule that reacts instantly in aqueous solution with substrates such as unsaturated fatty acids, reducing compounds such as reduced glutathione (GSH) and some water-soluble proteins such as uric acid, ascorbic acid, albumin, glucose and those rich in cysteine.

The biochemical reactions determine the formation of an excess of $\mathrm{H} 2 \mathrm{O} 2$, which, by spreading in the cells, activates numerous metabolic pathways. At the same time, the excess of $\mathrm{H} 2 \mathrm{O} 2$ is reduced by the intracellular antioxidant system represented mainly by the superoxide dismutase (SOD), catalase and glutathione 
(GSH) enzymes. ${ }^{37-40,41}$ Cellular antioxidant systems prevent the accumulation of $\mathrm{H} 2 \mathrm{O} 2$ and $\mathrm{O} 2$ by transforming them before they can damage cellular structures. GSH in the presence of $\mathrm{H} 2 \mathrm{O} 2$ is transformed into its disulfuric form by the enzyme GSH-peroxidase according to the reaction:

\section{$2 \mathrm{GSH}+\mathrm{H} 202=\mathrm{GSSG}+2 \mathrm{H} 2 \mathrm{O}$}

The oxidized glutathione by the action of the enzyme GSHreductase is made available in the reduced form:

$\mathrm{GSSG}+\mathrm{NADPH}+\mathrm{H}+=2 \mathrm{GSH}+\mathrm{NADP}+$

The mechanism described above protects membrane lipids from peroxidation as well as nucleic acids and endo-cellular proteins. ${ }^{31,36}$

It is shown that ozone generates free radicals only in an environment with a PH higher than 8 while with $\mathrm{PH}$ lower than 7.5 the ionic reaction mechanism intervenes, producing peroxides instead of radicals according to the ozonolysis reaction.

Ozone, in adequate quantities, has no undesirable effects, generating free radicals only at high concentrations and when antioxidative protection systems are exceeded. ${ }^{31,36,40}$

Therapy with oxygen-ozone mixtures rarely causes side effects. Side effects are mainly caused by errors in how the therapy is administered. A study carried out in Germany on 400,000 patients found the presence of undesirable effects in 7 cases on one million applications. This incidence corresponds to $0.0007 \% .{ }^{31} \mathrm{In}$ the implementation of therapy with $\mathrm{O} 2 \mathrm{O} 3$, it is fundamental to reaffirm the concept of useful administered dose, understood as the amount of mixture to the proper concentration and with the use of a correct administration technique, as indicated by the SIOOT protocols approved by the Ministry of Health. The effects that $\mathrm{O} 3$ exerts on the metabolism justifies the wide range of indications and therapeutic applications. Among the most renowned and here briefly reported are: the increase in glycolysis with a higher production of ATP, the stimulation of lipid metabolism, important effects on red blood cells (Bohr effect) and microcirculation, greater synthesis of 2,3 diphosphoglycerate, reduction of blood viscosity and platelet aggregation, a powerful static and bactericidal virus action. ${ }^{31,36}$

In the immune system, ozone determines an important immunomodulatory action with a reduction of immunoglobulins and immune complexes in the serum. Oxidizing agents such as hydrogen peroxide (H2O2) participate by stimulating the expression of proinflammatory cytokines by monocytes, macrophages and lymphocytes at the area of the lesion. Ozone exposure interferes with the synthesis and regulation of interferon $\alpha, \beta, \gamma$ (INF $\alpha-$ $\beta-\gamma$ ), tumor necrosis factor- $\alpha$ (TNF- $\alpha$ ), interleukin (IL) 1-2-4-6-810 , of granulopoietins (GM-CSF) and of the transforming growth factor $\beta$ (TGF- $\beta) .{ }^{31,36,39}$

The application of $\mathrm{O} 2 \mathrm{O} 3$ therapy produces the rapid remission of symptoms, reducing pain and inflammation in just a short time, returning the tendon, ligament and joint to their proper functions.

These beneficial effects are due to the inhibition of pro-inflammatory prostaglandin synthesis, the reduction of bradykinins and of algogenic compounds in the damaged tissue. The action of ozone produces the neutralization of reactive oxygen species (ROS) with greater stimulation of the local synthesis of antioxidant enzymes, the inhibition of proteinase release by macrophages and of polymorphonucleares and modulation for production, for anti-inflammatory purposes, of the pro-inflammatory cytokines. The oxidative stress caused by intense muscle activity produces the activation of nuclear transcription factor kappa B (NFKB) with a greater synthesis of
COX2, PGE2 and cytokines, pro-inflammatories (Figure 5). ${ }^{24,30,37,38}$

In tissues, moderate oxidative stress, caused by the ozone, is able to facilitate the repair and healing processes. The $\mathrm{O} 3$ induces, from the very beginning of the first phase of modelling, an increased expression of nuclear factor erythroid transcription factor 2 (Nrf2) and the synthesis of antioxidant response element (ARE) with greater production of antioxidant enzymes, including SOD, GSH, GSH-reductase, GSH-S transferase (gstr), Glutathione peroxidase (GPX), glutathione-s-transferase (GST), Catalase (CAT), emeoxigenase-1 ( HO-1), NADPH-quinone oxido-reductase (NQO1), Heat Shock Protein 70 kilodaltons (HSP70) and phase II enzymes, protecting cellular structures from acute and chronic oxidation damage. $24,31,36,39$

Oxygen and ozone therapy is carried out for the effectiveness demonstrated on vascular diseases, intervening on the activation of nuclear factor transcription, hypoxia-inducible factor- $1 \alpha$ (HIF-1 $\alpha)$ which can be stimulated by moderate stress. The effects on ozonized erythrocytes show better glycolysis and a greater increase in intra-Hypopic ATP and 2,3 diphosphoglycerate (2,3DPG), shifting the dissociation curve of $\mathrm{HbO} 2$ hemoglobin to the right, favoring a better oxygen release to tissues. ${ }^{24,36,40}$ Ozone induces an increase in erythrocyte deformability because it breaks the long fatty acid chains through controlled lipoperoxidation, facilitating the flow of red cells in the capillaries and avoiding the phenomenon of stacking in the vessels (Fahraeus-Lindquist effect) facilitating metabolic exchanges. ${ }^{36}$

Low-concentration ozone administration reduces overall blood viscosity and platelet aggregation as well as plasma fibrinogen, resulting in improved blood flow also due to increased nitric oxide synthesis and increased release at pre-capillary sphincters. ${ }^{36,39,40}$

Finally, ozone has a strong antibacterial and antiviral action with a powerful antioxidant action, improving the protective functions of cells.

\section{Conclusions}

The antiedema, anti-inflammatory, immunomodulatory and angiogenic properties make oxygen ozone therapy a suitable

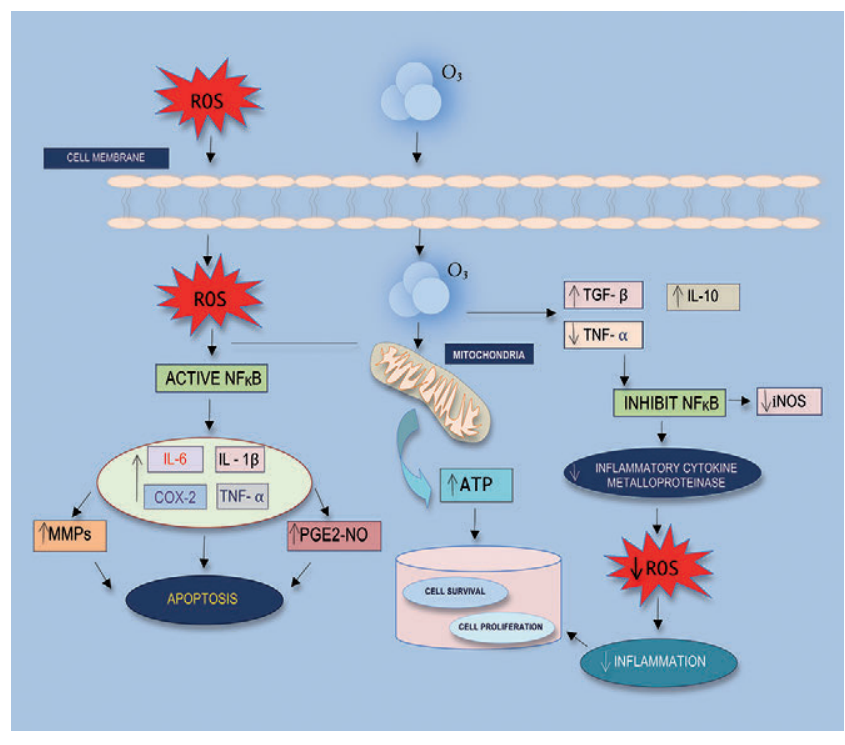

Figure 5. Ozone action. 
method in the application of all acute, chronic, degenerative and traumatic disorders of tendons and ligaments. The healing abilities have beneficial effects during all repair and healing process phases, creating the conditions for a shorter clinical course, the rapid remission of symptoms and the complete restitution of motor functions, without presenting any side effects.

\section{References}

1. INAIL I DISTURBI MUSCOLOSCHELETRICI LAVORATIVI - Enrico Occhipinti, Daniela Colombini, Diego Alhaique, Elisabetta Badellino, Alberto Baratti, Marisa Calamita, Angela Goggiamani, Doriano Magosso, Barbara Manfredi, Elena Mattace Raso, Giulia Ombuen, Ottorino Umberto Osnato, Adriano Ossicini, Adriano Papale, Tipolitografia INAIL Milano, aprile 2012 - ISBN 978-88-7484-227-8.

2. CLINICAL LECTURE ON CERTAIN CONDITIONS OF THE HAND AND ARM WHICH INTERFERE WITH THE PERFORMANCE OF PROFESSIONAL ACTS, ESPECIALLY PIANO-PLAYING. G. VIVIAN POORE, Physicianto UniversityCollegeHospital. THE BRITISH MEDICALJOURNAL - Feb. 26, 1887.

3. EPIDEMIOLOGIA DEI PROBLEMI MUSCOLOSCHELETRICI DEI MUSICISTI - F. CATALANO, M. CERUSO, S. PFANNER, A. ATZEI, P. TOS Riv Chir Mano Vol. 48 (2) 2011.

4. CORRELATION BETWEEN RISK FACTORS AND MUSCULOSKELETAL DISORDERS AMONG CLASSICAL MUSICIANS - Y. Kaufman-Cohen and N.Z. Ratzon Occupational Medicine 2011;61:90-95 Advance Access publication on 26 January 2011 doi:10.1093/occmed/kqq196.

5. IL POLLICE NEL MUSICISTA - G. GUIDI, S. PFANNER, M. CERUSO - Riv Chir Mano - Vol. 48 (2) 2011.

6. BERNSTEIN'S HERITAGE - DISCOVERING THE RIGHT QUESTIONS IN MOTOR CONTROL: MOVEMENTS (1929) - Anatol G. Fel'dman and Onno G. Meger - Motor Control, 1999,3, 105.134 1999 Human Kinetics Publishers, Inc.

7. COORDINATION OF BOWING AND FINGERING IN VIOLIN PLAYING - Andreas P. Baader, Oleg Kazennikov, Mario Wiesendanger - Cognitive Brain Research 23 (2005) 436-443.

8. BIMANUAL COORDINATION OF BOWING AND FINGERING IN VIOLINISTS-EFFECTS OF POSITION CHANGES AND STRING CHANGES - Oleg Kazennikov and Mario Wiesendanger - Published in "Motor Control 13(3): 297-309, 2009" which should be cited to refer to this work.

9. NEUROLOGICAL PRINCIPLES AND REHABILITATION OF ACTION DISORDERS: COMPUTATION, ANATOMY, AND PHYSIOLOGY (CAP) MODEL. Scott H. Frey, Leonardo Fogassi, Scott Grafton, Nathalie Picard, John C. Rothwell, Nicolas Schweighofer, Maurizio Corbetta, and Susan M. Fitzpatrick. Neurorehabilitation and Neural Repair Supplement to 25(5)65-20S CTheAuthor(s) 20II Reprints and permission: http://www. sagepub.com/journals Permissions. nav DOl: 10.117711545968311410940.

10. MUSCLE MOMENT-ARMS: A KEY ELEMENT IN MUSCLE-FORCE ESTIMATION David Ingram, Christoph Engelhardt, Alain Farron, Alexandre Terrier and Philippe Müllhaupt. Computer Methods in Biomechanics and Biomedical Engineering, 2015 Vol.18, No. 5, 506-513.

11. DAMPING ACTIONS OF THE NEUROMUSCULAR SYSTEM WITH INERTIAL LOADS: HUMAN FLEXOR POLLICIS LONGUS MUSCLE - DAVID C. LIN AND W. ZEV
RYMER. Department of Biomedical Engineering, Northwestern University; and The Sensory Motor Performance Program, Rehabilitation Institute of Chicago, Chicago, Illinois 60611 Received 4 November 1999; accepted in final form 17 November 2000 - The American Physiological Society - 0022-3077/01.

12. IN VIVO INVESTIGATION OF TENDON RESPONSES TO MECHANICAL LOADING K.M. HEINEMEIER AND M. KJAER INSTITUTE OF Sports Medicine, Bispebjerg Hospital and Centre for Healthy Ageing, Faculty of Health Sciences, University of Copenhagen - J Musculoskelet Neuronal Interact 2011;11(2):115-123.

13. MODELING AND SIMULATING THE DEFORMATION OF HUMAN SKELETAL MUSCLE BASED ON ANATOMY AND PHYSIOLOGY. Robson R. Lemos, Jon Rokne, Gladimir V.G. Baranoski, Yasuo Kawakami and Toshiyuki Kurihara Published online in Wiley InterScience (www.interscience. wiley.com). DOI: 10.1002/cav.83.

14. BIOMECHANICAL BEHAVIOR OF MUSCLE-TENDON COMPLEX DURING DYNAMIC HUMAN MOVEMENTS Senshi Fukashiro, Dean C. Hay and Akinori Nagano University of Tokyo; RIKEN, Japan - Journal of Applied Biomechanics, 2006;22:131-147. (C) 2006 Human Kinetics, Inc.

15. CONSTITUTIVE MODELING OF SKELETAL MUSCLE TISSUE WITH AN EXPLICIT STRAIN-ENERGY FUNCTION - G.M. Odegard and T.L. Haut Donahue, D.A. Morrow and K.R. Kaufman - Published in final edited form as: J Biomech Eng. 2008 December; 130(6):061017. doi:10.1115/ 1.3002766 .

16. PROFESSIONAL BALLET DANCERS' EXPERIENCE OF INJURY AND OSTEOPATHIC TREATMENT IN THE UK: A QUALITATIVE STUDY - Tobias Pollard-Smith, Oliver P. Thomson. Journal of Bodywork \& Movement Therapies (2017) 21, 148e156.

17. TENDON VASCULATURE IN HEALTH AND DISEASE HERBERT TEMPFER AND ANDREAS TRAWEGER Frontiers in Physiology - published: 18 November 2015 doi: 10.3389/fphys.2015.00330.

18. THE ROLE OF TENDON MICROCIRCULATION IN ACHILLES AND PATELLAR TENDINOPATHY - Karsten Knobloch -Published: 30 April 2008 - Journal of Orthopaedic Surgery and Research 2008, 3:18 - doi:10.1186/1749-799X-3-18.

19. THE VASCULATURE AND ITS ROLE IN THE DAMAGED AND HEALING TENDON - Steven A. Fenwick, Brian L. Hazleman and Graham P. Riley - Arthritis Res 2002, 4:252-260.

20. THE ACHILLES TENDON: FUNDAMENTAL PROPERTIES AND MECHANISMS GOVERNING HEALING - Benjamin R. Freedman Joshua A. Gordon Louis J. Soslowsky - Muscles, Ligaments and Tendons Journal 2014;4 (2):245-255.

21. MODELS FOR THE STUDY OF TENDINOPATHY - R.C. Dirks and S.J. Warden - J Musculoskelet Neuronal Interact 2011;11(2):141-149.

22. CHRONIC TENDINOPATHY TISSUE PATHOLOGY, PAIN MECHANISMS, AND ETIOLOGY WITH A SPECIAL FOCUS ON INFLAMMATION - U. Fredberg, K. StengaardPedersen - Scand J Med Sci Sports 2008;18:3-15 - DOI: 10.1111/j.1600-0838.2007.00746.x.

24.MECHANISMS OF ACTION INVOLVED IN OZONE THERAPY: IS HEALING INDUCED VIA A MILD OXIDATIVE STRESS? - Masaru Sagai and Velio Bocci - Medical Gas Research 2011;1:29 - doi:10.1186/2045-9912-1-29.

25. IMPACT OF OXIDATIVE STRESS ON EXERCISING SKELETAL MUSCLE - Peter Steinbacher and Peter Eckl Biomolecules 2015;5,356-377; doi:10.3390/biom5020356. 
26. REDOX CHARACTERIZATION OF FUNCTIONING SKELETAL MUSCLE - Li Zuo and Benjamin K. Pannell Front. Physiol. 6:338. doi: 10.3389/fphys.2015.00338

27. SYSTEMIC CYTOKINE RESPONSE FOLLOWING EXERCISE-INDUCED MUSCLE DAMAGE IN HUMANS Anastassios Philippou, Gregory Bogdanis, Maria Maridaki, Antonis Halapas, Antigone Sourla and Michael Koutsilieris, Clin Chem Lab Med 2009;47(6):777-782 2009 by Walter de Gruyter - Berlin • New York. DOI 10.1515/CCLM.2009.163.

28. CHANGES IN INFLAMMATORY MEDIATORS FOLLOWING ECCENTRIC EXERCISE OF THE ELBOW FLEXORS - Lisa Hirose, Kazunori Nosaka, Michael Newton, Andrew Laveder, Masumi Kano, Jonathan Peake, and Katsuhiko Suzuki - Eccentric exercise and cytokines - Exerc Immunol Rev. 2004;10:75-90.

29. TENDON INJURY AND TENDINOPATHY: HEALING AND REPAIR - BY PANKAJ SHARMA, MRCS, AND NICOLA MAFFULLI, MD, MS, PHD, FRCS(ORTH) - THE JOURNAL OF BONE \& JOINT SURGERY · JBJS.ORG VOLUME 87-A · NUMBER 1 · JANUARY 2005 doi:10.2106/JBJS.D.01850.

30. BIOLOGY OF TENDON INJURY: HEALING, MODELING AND REMODELING P. Sharma and N. Maffulli - J Musculoskelet Neuronal Interact 2006;6(2):181-190.

31. SCIENTIFIC AND MEDICAL ASPECTS OF OZONE THERAPY. STATE OF THE ART - Velio Alvaro Bocci Rivista Italiana di Ossigeno-Ozonoterapia 5:93-104,2006 doi:10.1016/ j.arcmed.2005.08.006.

32. NITRIC OXIDE ENHANCES COLLAGEN SYNTHESIS IN CULTURED HUMAN TENDON CELLS - Wei Xia, Zoltan Szomor, Yao Wang, George A.C. Murrell - Published online 21 November 2005 in Wiley InterScience - DOI 10.1002/ jor.20060.

33. TENDON DEVELOPMENT AND MUSCULOSKELETAL ASSEMBLY: EMERGING ROLES FOR THE EXTRACELLULAR MATRIX - Arul Subramanian and Thomas F.
Schilling - (C) 2015. Published by The Company of Biologists Ltd | Development (2015) 142, 4191-4204 doi:10.1242 /dev.114777.

34. TENDON AND LIGAMENT TISSUE ENGINEERING, HEALING AND REGENERATIVE MEDICINE - ALI MOSHIRI AND AHMAD ORYAN - Sports Med Doping Stud 2013 - Volume 3 • Issue 2 • 1000126 - http://dx.doi.org/ 10.4172/2161-0673.1000126.

35. GROWTH FACTORS FOR ROTATOR CUFF REPAIR LAWRENCE V. GULOTTA, SCOTT A. RODEO - Clin Sports Med 28 (2009) 13-23 doi:10.1016/j.csm.2008. 09.002.

36. OSSIGENO-OZONO TERAPIA - L. Valdenassi, M. Franzini, P. Richelmi, F. Berte - Atti del Convegno della Sezione SIMFER di Riabilitazione Ambulatoriale «Passaggio Lombo-sacrale e Sacro-iliache: progetto e programmi riabilitativi multidisciplinari» a cura di M. Monticone Pavia, 22 Novembre 2003.

37. REDOX REGULATION OF MUSCLE ADAPTATIONS TO CONTRACTILE ACTIVITY AND AGING - J Appl Physiol 119: 163-171, 2015. - First published March 19, 2015; doi:10.1152/japplphysiol.00760.2014.

38. ACUTE EXERCISE AND OXIDATIVE STRESS: A 30 YEAR HISTORY - Kelsey Fisher-Wellman and Richard J Bloomer - Dynamic Medicine 2009, 8:1 doi:10.1186/14765918-8-1.

39. OXYGEN-OZONE THERAPY: PARADOXICAL STIMULATION OF OZONE - Luigi Valdenassi, Marianno Franzini, Vincenzo Simonetti, Giovanni Ricevuti, Oxygen-Ozone Therapy Scientific Society, Gorle (BG); Department of Internal Medicine and Medical Therapy, University of Pavia; Kaos non profit Association, Caselle Torinese (TO); Department of Internal Medicine, University of Pavia, Italy.

40. OXYGEN/OZONE AS A MEDICAL GAS MIXTURE. A CRITICAL EVALUATION OF THE VARIOUS METHODS CLARIFIES POSITIVE AND NEGATIVE ASPECTS - Velio Bocci, Iacopo Zanardi and Valter Travagli. Medical Gas Research 2011, 1:6 - doi:10.1186/2045-9912-1-6. 